\title{
Expression of aquaporin-5 in and fluid secretion from immortalized human salivary gland ductal cells by treatment with 5-aza-2'-deoxycytidine: a possibility for improvement of xerostomia in patients with Sjögren's syndrome
}

\author{
Katsumi Motegi, Masayuki Azuma, Tetsuya Tamatani, Yuki Ashida and Mitsunobu Sato \\ Second Department of Oral and Maxillofacial Surgery, Tokushima University School of Dentistry, \\ Tokushima, Japan
}

\begin{abstract}
The aim of the present study was to investigate the possibility that ductal cells, which preferentially survive and/ or proliferate in Sjögren's syndrome (SS) salivary glands of patients with SS, could acquire the functional expression of membrane water channel aquaporin-5 (AQP5). Thus, in this study, we demonstrate that an immortalized normal human salivary gland ductal cell (NS-SV-DC) line, lacking the expression of AQP5, acquires AQP5 gene expression in response to treatment with 5-aza-2'-deoxycytidine (5-Aza-CdR), a DNA demethylating agent. Confocal microscopic analysis revealed the localization of AQP5 expression mainly at the apical and lateral sides of the plasma membrane. The expressed AQP5 protein was functionally active because AQP5 expression resulted in a significant increase in the osmotically directed net fluid rate across monolayers of NS-SV-DC cells. By the analysis of bisulfite sequencing of CpG islands in the AQP5 promoter, hypermethylation within the consensus Sp1-binding sites was commonly observed in parental cell clones, whereas demethylation at the CGs, one in the second consensus Sp1 element and the other outside of the third consensus Sp1 element in the AQP5 promoter, was detected in NS-SV-DC cells after treatment with 5-Aza-CdR. By analyzing the luciferase activity of transfected AQP5 promoter vectors, it became evident that demethylation at the CGs cooperatively functions between these two sites to induce AQP5 expression. Our data, therefore, suggest that treatment of ductal cells with 5-Aza-CdR could result in the expression of the AQP5 gene, thereby leading to increased fluid secretion from ductal cells in SS salivary glands.
\end{abstract}

Laboratory Investigation (2005) 85, 342-353, advance online publication, 10 January 2005; doi:10.1038/labinvest.3700234

Keywords: aquaporin-5; 5-aza-2'-deoxycytidine; salivary gland ductal cells; salivary gland acinar cells; methylation

Sjögren's syndrome (SS), one of the most common rheumatic diseases, ${ }^{1}$ is characterized by the eventual total replacement of the acinar structure by marked lymphocytic infiltrates in salivary and lacrimal glands. ${ }^{2}$ As a general clinical feature, patients with SS suffer from progressive dryness of the mouth and eyes due to insufficient salivary and

Correspondence: Dr M Azuma, DDS, PhD, Second Department of Oral and Maxillofacial Surgery, Tokushima University School of Dentistry, 3 Kuramoto-cho, Tokushima 770-8504, Japan.

E-mail: azumasa@dent.tokushima-u.ac.jp

Received 9 September 2004; revised 9 November 2004; accepted 30 November 2004; published online 10 January 2005 lacrimal secretions. Other symptoms associated with SS, especially in patients complaining of dry mouth, include diffuse oral burning, dysphagia, dysgeusia, and hoarseness. Thus, for the clinical improvement of patients with SS, development of a novel therapeutic modality to enhance salivary secretion would be inevitable.

Histopathologic features of SS salivary glands include: (a) the eventual total replacement of the acinar structure by marked lymphocytic infiltrate and (b) the occurrence of various changes in the ductal structure within infiltrated areas, such as metaplasia, hyperplasia, thinning of ductal layer, or oncocytic change, and in some cases the formation 
of epimyoepithelial islands arising from ductal proliferation. ${ }^{2,3}$ Accordingly, surviving and/or proliferating ductal cells in SS salivary glands may be regarded as one of the possible sources for the improvement of salivary secretion.

Aquaporins (AQPs) are specific water channels that allow the rapid transcellular movement of water in response to osmotic/hydrostatic pressure gradients. ${ }^{4}$ One of the AQPs, AQP5, cloned from rat submandibular glands, is present in the watertransporting epithelia of the lacrimal gland, trachea, eye, lung, and salivary glands. ${ }^{5}$ In human salivary glands, AQP5 has been topographically localized to the apical membranes of acinar cells but not to those of ductal cells. ${ }^{6}$ It functions to stimulate the outflow of water into the acinar lumen. In fact, a reduction in salivary gland secretion has been shown in mice harboring the mutant AQP5 channel. ${ }^{7}$ Another AQP, AQP3, has been reported to be localized to the basolateral surface of acinar cells, where it allows the inflow of water into those cells. ${ }^{8}$ Thus, it is likely that the functional presence of both AQP5 and AQP3 in acinar cells may, at least in part, be responsible for the normal fluid outflow. Based on these findings, it might be feasible to hypothesize that ductal cells could acquire the ability to secrete salivary fluid when they express these AQPs, especially AQP5.

Recent studies have indicated that hypermethylation of $\mathrm{CpG}$ islands within the promoter and $5^{\prime}$ regions of genes is an important epigenetic mechanism for suppressing gene expression. ${ }^{9-11}$ DNA hypermethylation may directly affect the basal transcriptional machinery by altering the DNA secondary structure and inducing chromosome remodeling through the methyl-group binding proteins and histone deacetylase, thereby leading to transcriptional repression. ${ }^{12}$ Treatment with a DNA demethylating agent, such as 5-aza-2'-deoxycytidine (5-Aza-CdR), reactivates many genes that have been inactivated by hypermethylation. ${ }^{13-17}$ However, although the AQP5 promoter contains three consensus Sp1-binding sites, ${ }^{18}$ the relationship between the methylation status of the AQP5 promoter and the gene expression has not yet been examined in human salivary gland cells.

In the present study, we examined the mechanism by which immortalized normal human salivary gland ductal cells, expressing AQP3 but not AQP5, acquire the ability to express AQP5 and secrete fluid in response to 5-Aza-CdR. The results of the present study show a causal relation between hypomethylation of the AQP5 promoter and AQP5 gene expression in ductal cells of the human salivary gland. We also demonstrate that demethylation at CG sites, both in the second consensus Sp1-binding site and outside of the third consensus Sp1-binding site of the AQP5 promoter region, may directly activate the transcription of the AQP5 gene in ductal cells.

\section{Materials and methods}

\section{Cells and Media}

The characteristics of immortalized normal human salivary gland ductal (NS-SV-DC) and acinar (NSSV-AC) cell clones have already been described in detail elsewhere. ${ }^{19,20}$ In brief, normal human salivary gland cells obtained from explants of a submandibular gland with no histopathologic disorders were grown in serum-free keratinocyte medium (SFKM) (Gibco BRL, Grand Island, NY, USA). After transfection with SV40 origin-defective mutant DNA, transfected cells were cloned by the limiting dilution method. In this study, we used two of four cell clones having distinct phenotypes: one with a ductal phenotype and one with an acinar phenotype. They were employed in the present study for 150 passages from the beginning of the culture. Even after more than 150 passages, neither anchorage-independent colonies in soft agar nor tumorigenicity in nude mice were detected in either clone, suggesting that no apparent phenotypic changes occurred in these immortalized cell clones. These cell clones were cultured at $37^{\circ} \mathrm{C}$ in SFKM in an incubator with an atmosphere containing $5 \% \mathrm{CO}_{2}$.

\section{Growth Assay}

Cells $\left(1 \times 10^{4} /\right.$ well $)$ were grown in 96-well plates (Falcon, Oxnard, CA, USA) in SFKM in the presence of 5-Aza-CdR at concentrations of $0,500 \mathrm{nM}, 1 \mu \mathrm{M}$, and $2 \mu \mathrm{M}$. After the appropriate incubation periods, $10 \mu \mathrm{l}$ of a $5 \mathrm{mg} / \mathrm{ml}$ preparation of 3-(4,5-dimethylthizol-2-yl)-2,5-diphenyltetrazolium bromide (MTT) was added to each well and incubation was continued for $4 \mathrm{~h}$. The reaction was terminated by the addition of $100 \mu \mathrm{l}$ of a solution of $0.04 \mathrm{~N} \mathrm{HCl}$ in isopropanol, and the absorbance was measured with a Titertek spectrophotometer (Flow, Irvine, UK) at $570 \mathrm{~nm}$ with a reference wavelength of $630 \mathrm{~nm}$. All assays were run in triplicate.

\section{RNA Isolation, RT-PCR, and Quantitative Real-Time PCR}

Total cellular RNA was isolated at $0,48,72,96$, and $120 \mathrm{~h}$ after NS-SV-DC cells were treated with 5-AzaCdR at a concentration of $2 \mu \mathrm{M}$ using TRIzol reagent (Invitrogen, Carlsbad, CA, USA). As a positive control for AQP5, normal human submandibular gland tissues were used. This submandibular gland was obtained from surgery to treat sialolithiasis. The cDNA was synthesized from $10 \mu \mathrm{g}$ of total RNA using the Advantage cDNA PCR Kit (Clontech, Palo Alto, CA, USA). The sense and antisense primers for AQP5, AQP3, and glyceraldehyde-3-dehydrogenase (GAPDH), respectively, were

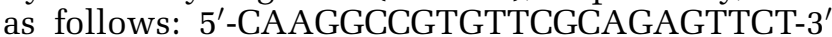


and 5'-TCTTCCGCTCTTCCCGCTGCTCC-3', 5'-CCT TTGGCTTTGCTGTCACTC-3' and $5^{\prime}$-ACGGGGTTG TTGTAGGGGTCA-3' ${ }^{\prime}$, and $5^{\prime}$-ACGCATTTGGCTGTA TTGGG-3' and 5'-TGATTTTGGAGGGATCTCGC-3'. The PCR reactions were conducted in a DNA Thermal Cycler model TP-3000 (Takara, Otsu, Japan). After $1 \mathrm{~min}$ of denaturation at $94^{\circ} \mathrm{C}, 35$ cycles of PCR were performed $\left(94^{\circ} \mathrm{C}\right.$ for $30 \mathrm{~s} / 68^{\circ} \mathrm{C}$ for $2 \mathrm{~min}$ ), followed by a final 3 -min extension at $72{ }^{\circ} \mathrm{C}$. For quantitative real-time PCR, equal aliquots $(2 \mu \mathrm{l})$ of cDNA were amplified according to the manufacturer's TaqMan universal (50 $\mu$ l) PCR master mix protocol using reverse transcriptase-polymerase chain reaction (RT-PCR) ABI PRISM 7000 (Applied Biosystems Japan Ltd., Tokyo, Japan). A primer set and TaqMan probe used for AQP5 PCR were designed as follows: primers, 5'-CTGCGGTGGTCAT GAATCG-3' and 5'-TTGATGATGGCCACACGCT-3'; probe, 5'-FAM-CCTACCCAGAAAACCCAGTGAGC GG-MGB-3'. The data were normalized using RT PCR GAPDH primers (Applied Biosystems).

\section{Western Blot Analysis of AQP5}

Crude plasma membranes were prepared from 5Aza-CdR-treated or untreated NS-SV-DC cells using Mem-PER Eukaryotic Membrane Protein Extraction Reagent Kit (Pierce Biotechnology, Rockford, IL, USA) according to the manufacturer's instructions. Crude membrane extract containing $20 \mu \mathrm{g}$ of protein was subjected to electrophoresis in $15 \%$ SDSpolyacrylamide gel electrophoresis, then transferred to a nitrocellulose membrane. The membranes were blocked with $3 \%$ bovine serum albumin and incubated with either a goat anti-human AQP5 antibody (Santa Cruz Biotechnology, Inc., Santa Cruz, CA, USA) or a rabbit anti-human $\beta$-actin antibody (Abcam Ltd, Cambridge, UK). After intervening rinses with PBS, the antibody was detected using a chemiluminescence Western Blotting kit (Amersham, Tokyo, Japan) according to the manufacturer's instructions. The specificity of the AQP5 antibody was determined by using a blocking peptide, that is, the AQP5 antibody was mixed with AQP5 blocking peptide (Santa Cruz Biotechnology) at the ratio of $25: 1$, followed by incubation for $2 \mathrm{~h}$ at room temperature. The mixture was then used as a first antibody for the detection of AQP5 protein. In addition, the crossreactivity of the anti-AQP5 antibody with the anti-AQP3 antibody was also examined using blocking peptide for either AQP5 or AQP3 (Santa Cruz Biotechnology) by Western blot analysis.

\section{Net Fluid Secretion Rate Measurement}

The net fluid secretion rates in the control NS-SVDC cells and in the cells treated with either 5-AzaCdR $(2 \mu \mathrm{M})$ or histone deacetylase inhibitor suberoylanilide hydroxamic acid (SAHA) (500 nM) for $48 \mathrm{~h}$ were measured using a modified form of the original method. ${ }^{21}$ In brief, NS-SV-DC cells were grown to confluence on six-well Transwell-Col culture chambers (Coster, Cambridge, MA, USA). The apical fluid was replaced by $0.4 \mathrm{ml}$ of hyperosmotic medium (400 mOsm, ie $100 \mathrm{mM}$ sucrose in medium), and the medium from the basolateral chamber was replaced by fresh isosmotic medium (300 mOsm). After $4 \mathrm{~h}$, the liquid on the apical side was collected, its volume measured with a calibrated pipette, and the net fluid secretion rate was determined as described previously. ${ }^{22}$

\section{Localization of AQP5 Protein by Fluorescence Staining and Confocal Laser Microscopy}

Cells grown on plain coverglasses were washed with PBS three times, fixed in liquid containing acetone and methanol at the ratio of $6: 4$ at $4^{\circ} \mathrm{C}$ for $10 \mathrm{~min}$ and incubated for $1 \mathrm{~h}$ at $37^{\circ} \mathrm{C}$ with goat anti-human AQP5 antibody (Santa Cruz) at a dilution of 100. After three rinses with PBS containing 1\% bovine serum albumin, the cells were incubated for $1 \mathrm{~h}$ with fluorescence-conjugated donkey anti-goat IgG (1:50 dilution, Rockland, Gilbertsville, PA, USA). Coverglasses were mounted with $\mathbf{8 0} \%$ glycerol in PBS for confocal laser microscopy. Mounted coverglasses were examined with a TSC4D confocal laser microscope (Leica Microsystems AG, Wetzlar, Germany). Cells were optically sectioned in $x y$ and $x z$ planes.

\section{Isolation of Genomic DNA from 5-Aza-CdR-Treated or Untreated NS-SV-DC Cells}

The genomic DNA was isolated from NS-SV-DC cells treated with or without 5-Aza-CdR $(2 \mu \mathrm{M})$ using Promega's Wizard DNA isolation kit (Madison, WI, USA) according to the manufacturer's instructions.

\section{Bisulfite Sequencing}

In all, $2 \mu \mathrm{g}$ of DNA from NS-SV-DC cells treated with or without 5-Aza-CdR was modified by sodium bisulfite as described previously. ${ }^{23}$ The modified DNA was amplified with primers (forward primer, 5'-GGGAATTTCGGTTTGGGAGA-3'; reverse primer, $5^{\prime}$-CCCGTCCGAACCACGTAAC-3'). PCR reactions were performed as follows: $94^{\circ} \mathrm{C}$ for $1 \mathrm{~min}$; 35 cycles of $94^{\circ} \mathrm{C}$ for $30 \mathrm{~s}$ and $68^{\circ} \mathrm{C}$ for $2 \mathrm{~min}$; and then $72^{\circ} \mathrm{C}$ for $3 \mathrm{~min}$ at the end. The PCR products were gel extracted (Qiagen, Valencia, CA, USA) and ligated into a plasmid vector, pCR2.1-TOPO, by using the TA cloning system (Invitrogen). Plasmid-transformed bacteria TOP10 F' was cultured overnight, after which the plasmid DNA was isolated (Qiagen). Five separate clones from each treated or untreated cell clone were chosen for sequence analysis. 
Transient Transfection and Measurement of Relative Luciferase Activity

The human wild-type AQP5 promoter luciferase fusion plasmid, pC3-Luc, was made from a 575-bp genomic fragment of the AQP5 promoter containing the transcriptional start site subcloned into the luciferase reporter vector, pGL3Basic. pC3-Luc contains three Sp1 sites, termed Sp1-1, Sp1-2, and Sp1-3, including 43 CGs upstream from the transcriptional start site. In pC3-Luc, all CGs in the Sp1-1 site (1st CG), Sp1-2 site (23rd and 24th CGs), the 31st CG, and the Sp1-3 site (33rd CG) are methylated. pD3 contains unmethylated CG at the 24th CG and methylated CGs at the 1st, 23rd, 31st, and 33rd CGs. pD5 contains unmethylated CGs at the 24th and 31st CGs and methylated CGs at the 1st, 23rd, and 33rd CGs. pD7 contains unmethylated CG at the 31st CG and methylated CGs at the 1st, 23rd, 24th, and 33rd CGs. NS-SV-DC cells transfected with indicated AQP5-luciferase vectors were harvested for $24 \mathrm{~h}$ to analyze the luciferase activity (Lumat LB 9507; PerkinElmer Life Sciences, Berthold Technologies, Bad Wildbad, Germany). The luciferase activity was normalized for the amount of protein in the cell lysate. All luciferase assays were carried out in triplicate.

\section{Results}

\section{Effects of 5-Aza-CdR on Cell Growth}

The growth kinetics of NS-SV-DC cells treated with various concentrations of 5-Aza-CdR were investigated by MTT assay for up to 7 days. As shown in Figure 1, no remarkable cytotoxicity was observed when NS-SV-DC cells were treated with $500 \mathrm{nM}$, $1 \mu \mathrm{M}$, or $2 \mu \mathrm{M}$ of 5 -Aza-CdR. Thus, we selected a concentration of $2 \mu \mathrm{M}$ for the following experiments.

\section{AQP5 Gene Expression in NS-SV-AC and NS-SV-DC Cells}

RT-PCR was used to examine AQP5 mRNA expression in immortalized NS-SV-DC and NS-SV-AC cell lines. As shown in Figure 2, although both normal human salivary gland tissues employed as a positive control and acinar (NS-SV-AC) cells apparently expressed AQP5 mRNA (739bp); and ductal (NSSV-DC) cells lacked the expression of AQP5 mRNA. Since expression of AQP3 is important for the acinar cells' fluid-secreting function, ${ }^{8}$ we examined the expression of AQP3 mRNA in NS-SV-AC and NSSV-DC cells. Figure 2 shows that acinar cells as well as ductal cells expressed AQP3 mRNA (372 bp) at a similar expression level. In the above RT-PCR experiments, equal loading of RNA samples was demonstrated by measurement of the housekeeping gene GAPDH.

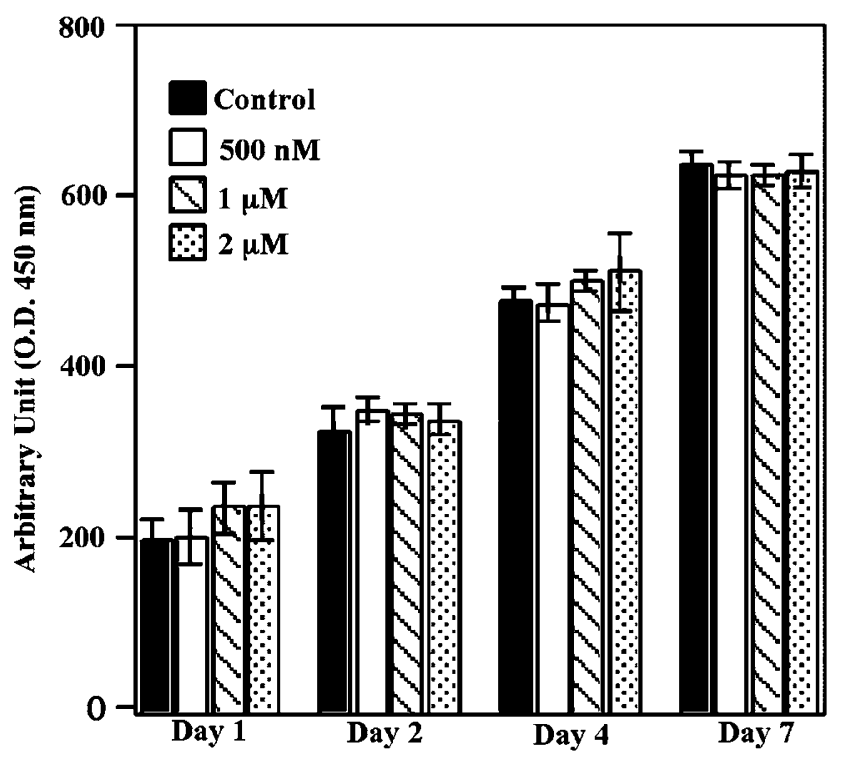

Figure 1 Growth properties of NS-SV-DC cells cultured in the presence or absence of 5 -Aza-CdR. Cells $\left(1 \times 10^{4} /\right.$ well $)$ were grown in 96-well plates in medium supplemented with 5-AzaCdR ( $500 \mathrm{nM}, 1 \mu \mathrm{M}$, or $2 \mu \mathrm{M}$ ) for up to 7 days. Viable cells were estimated by MTT assay. No significant suppression of cell growth was detected in any of the concentrations of 5-Aza-CdR.

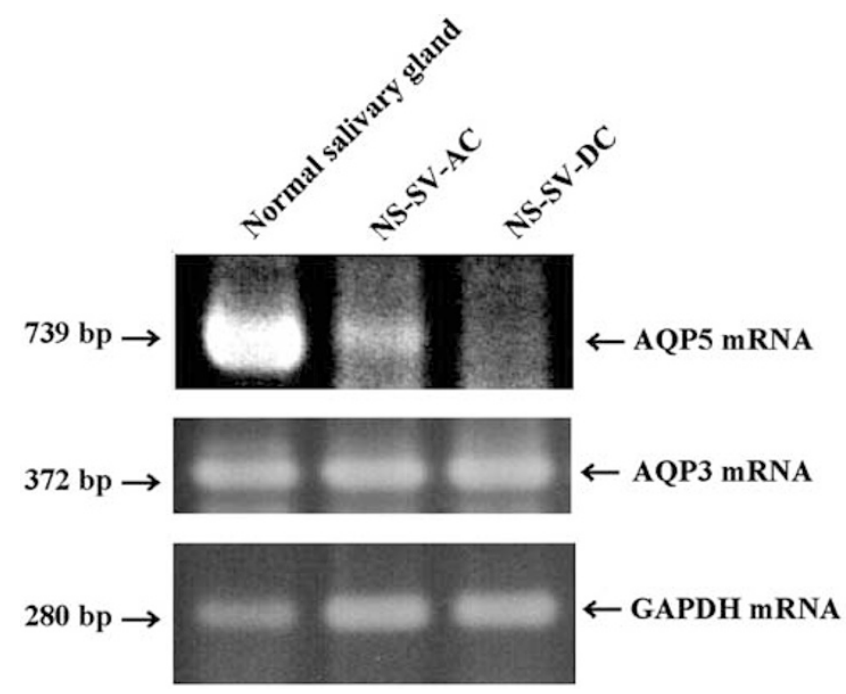

Figure 2 RT-PCR analysis for the expression of AQP5 and AQP3 mRNA in NS-SV-AC acinar and NS-SV-DC ductal cells. Normal human salivary gland tissues were used as a positive control. As expected, normal salivary gland tissues and NS-SV-AC acinar cells expressed AQP5 mRNA (739bp) and AQP3 mRNA (372 bp). However, although NS-SV-DC ductal cells expressed AQP3 mRNA, AQP5 mRNA was not detected. Equal loading of RNA samples was demonstrated for the housekeeping gene GAPDH.

\section{Induction of AQP5 mRNA and Protein in NS-SV-DC in Response to 5-Aza-CdR}

Time course analysis was used to investigate the induction of AQP5 mRNA expression in NS-SV-DC cells by treatment with a demethylating agent, 5 -Aza-CdR. After $48 \mathrm{~h}$ of 5-Aza-CdR $(2 \mu \mathrm{M})$ treatment, a significant increase in the AQP5 mRNA 


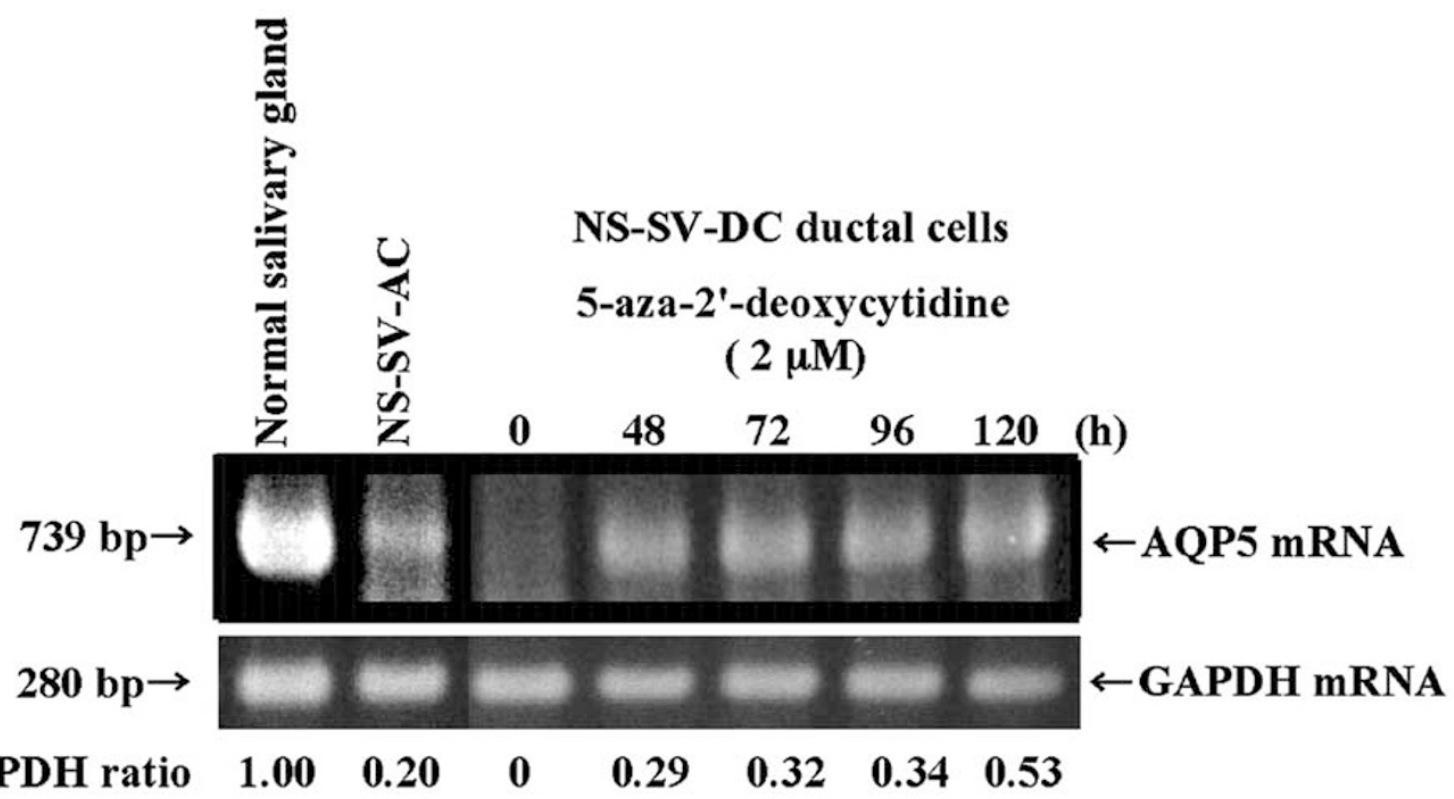

\section{$\begin{array}{llllllll}\text { AQP5/GAPDH ratio } & 1.00 & 0.20 & 0 & 0.29 & 0.32 & 0.34 & 0.53\end{array}$}

Figure 3 RT-PCR analysis for the expression of AQP5 mRNA in 5-Aza-CdR-treated NS-SV-DC ductal cells. NS-SV-DC cells were treated with 5-Aza-CdR $(2 \mu \mathrm{M})$ for $48,72,96$, or $120 \mathrm{~h}$. cDNA was prepared as described in Materials and methods. RT-PCR was performed using sense and antisense primers specific for AQP5 and GAPDH. Consistent expression of AQP5 mRNA (739 bp) was detected for up to $120 \mathrm{~h}$ examined. GAPDH mRNA was used as an equal loading of RNA samples. Densitometric analysis (AQP5/GAPDH mRNA ratio) revealed the expression level of AQP5 mRNA in 5-Aza-CdR-treated NS-SV-DC cells relative to that of normal salivary gland.

level was detected in NS-SV-DC cells (Figure 3). This high level continued for up to $120 \mathrm{~h}$. Densitometric analysis (NIH Image software, ver. 1.63) revealed that the expression level of AQP5/GAPDH mRNA in 5-Aza-CdR-treated NS-SV-DC at each time point relative to that of normal salivary gland was as follows: 0 at $0 \mathrm{~h}, 0.29$ at $48 \mathrm{~h}, 0.32$ at $72 \mathrm{~h}, 0.34$ at $96 \mathrm{~h}$, and 0.53 at $120 \mathrm{~h}$. Quantitative RT-PCR was employed to further examine the expression levels of AQP5 mRNA by 5-Aza-CdR in NS-SV-DC cells. As shown in Figure 4, a statistically significant increase in AQP5 mRNA expression was detected after treatment with 5-Aza-CdR: in NS-SV-AC acinar cells, the expression level of AQP5 mRNA was near the $10 \%$ of that of normal salivary gland tissues, and with the time of 5-Aza-CdR treatment, NS-SV-DC expressed an amount of AQP5 mRNA almost equal to that in salivary gland tissues. To detect the production of AQP5 protein, crude plasma membranes were subjected to Western blot analysis during preparation. As can be seen in Figure 5, AQP5 expression was significantly augmented by the 5-Aza-CdR treatment. Densitometric analysis demonstrated that the expression level of AQP5/ $\beta$ actin in 5-Aza-CdR-treated NS-SV-DC at each time point relative to that of normal salivary gland was as follows: 0 at $0 \mathrm{~h}, 0.24$ at $48 \mathrm{~h}, 0.25$ at $72 \mathrm{~h}, 0.26$ at $96 \mathrm{~h}$, and 0.78 at $120 \mathrm{~h}$. These values were similar to those of quantitative real-time RT-PCR analysis. When the AQP5 antibody was used after the mixture with blocking peptide, the band corresponding to AQP5 disappeared, suggesting that this AQP5 antibody was specific to AQP5 protein (data not shown).
Also, the crossreactivity of the anti-AQP5 antibody with the anti-AQP3 antibody was examined by Western blot analysis, but no crossreactivity was recognized (data not shown).

\section{Distribution of AQP5 in 5-Aza-CdR-Treated NS-SV-DC Cells}

In an effort to examine the localization of AQP5 in 5-Aza-CdR-treated NS-SV-DC cells, we used confocal laser microscopy. Untreated NS-SV-DC cells showed no AQP5 expression (Figure 6A, a). However, when NS-SV-DC cells were treated with $2 \mu \mathrm{M} 5$-AzaCdR for $72 \mathrm{~h}$, AQP5 protein was highly expressed (Figure 6A, b). As shown in Figure 6B, serial optical sections of $0.7 \mu \mathrm{m}$ thickness were made from the apical surface (i) toward the basal surface (xii). AQP5 expression in these cells was detected at both the apical and lateral sides of the plasma membrane.

\section{Fluid Secretion Rate in 5-Aza-CdR-Treated NS-SV-DC Cells}

To determine whether or not the AQP5 expressed in 5-Aza-CdR-treated NS-SV-DC cells affected their water permeability, we measured the transepithelial net fluid secretion rate in NS-SV-DC after treatment with 5-Aza-CdR. The net movement of fluid across NS-SV-DC cells was measured in the presence of a transepithelial (apical $>$ basal) osmotic gradient. After 4-h incubation, the net fluid secretion rate of control NS-SV-DC cells was approximately 3.8 
$\mu \mathrm{l} / \mathrm{cm}^{2}$ per hour. However, in 5-Aza-CdR-treated NS-SV-DC cells the net fluid secretion rate across the treated cells was significantly increased

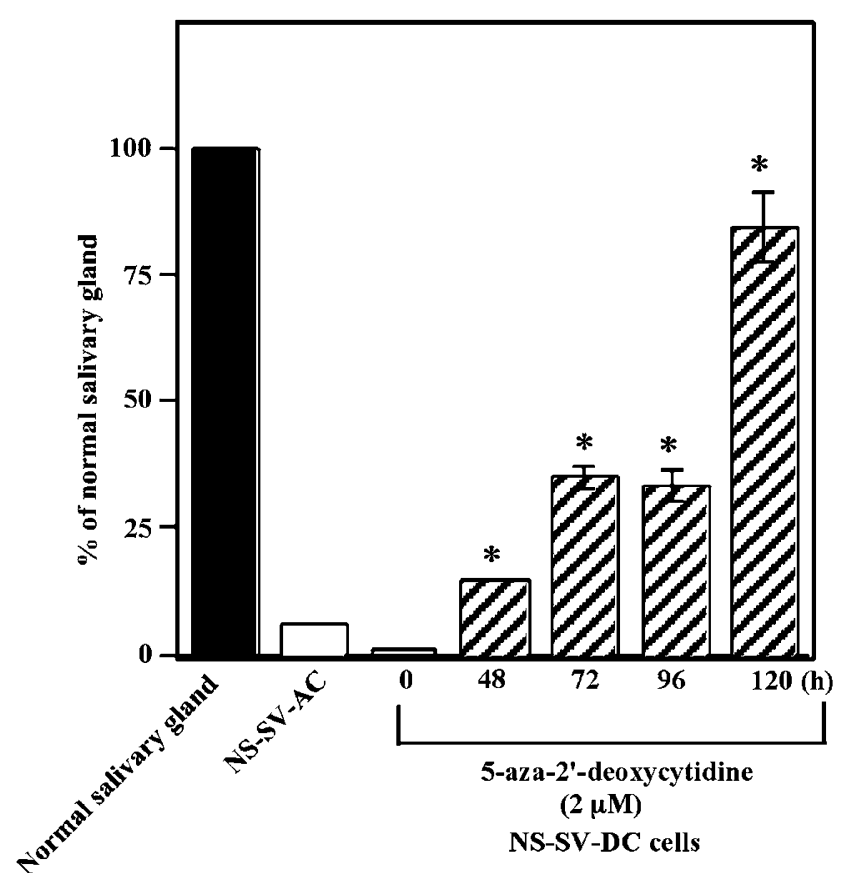

Figure 4 Steady-state levels of AQP5 mRNA measured using quantitative real-time PCR. The primers and probes used for these experiments are found in Materials and methods. Relative quantification of gene expression was performed as described by the manufacturer by using GAPDH mRNA as an internal standard. Induced expression levels of AQP5 mRNA in NS-SV-DC cells were compared with the expression level $(100 \%)$ in normal salivary gland tissues. Each bar represents at least three separate mRNA isolations performed in duplicate. NS-SV-AC acinar cells expressed a relatively small amount of AQP5 mRNA as compared to normal salivary gland tissues. 5-Aza-CdR-treated NS-SV-DC ductal cells acquired large amounts of AQP5 mRNA at $120 \mathrm{~h}$ after treatment. * Statistically significant at $P<0.05$ (Mann-Whitney $U$-test). $\left(7.0 \mu \mathrm{l} / \mathrm{cm}^{2}\right.$ per hour; Figure 7$)$, demonstrating that the expressed AQP5 was functional. To rule out the possibility that the increase in net fluid secretion from 5-Aza-CdR-treated NS-SV-DC ductal cells may be a nonspecific event, we examined the effect of a histone deacetylase inhibitor, SAHA, which is known as an activator of the human p21 gene promoter. ${ }^{24}$ SAHA treatment of NS-SV-DC ductal cells had no significant effect on the enhancement of fluid secretion under the same experimental conditions (data not shown).

\section{Hypermethylation of CpG Island of AQP5 Promoter in NS-SV-DC Cells}

To identify the methylation pattern within the $\mathrm{CpG}$ island of the AQP5 promoter in NS-SV-DC cells after treatment with or without 5-Aza-CdR, PCR products were subcloned into the vector pCR2.1-TOPO, and each of five separate subclones was sequenced. By analyzing the sequence of the human AQP5 promoter, three Sp1-binding sites were identified in this CpG island (Figure 8a). Figure $8 \mathrm{~b}$ shows the methylation status for all CGs in the tested CpG island (43 CGs) between -406 to +1 positions relative to the transcriptional start site of the AQP5 promoter. In the untreated NS-SV-DC cells, the CGs of Sp1-binding sites and their boundaries are heavily methylated (from the 23rd to 33rd CGs) (Figure 8b), while the 24th CG in the second Sp1binding site and the 31st CG around the third Sp1-binding site were unmethylated in the 5-AzaCdR-treated NS-SV-DC cells (Figure 8b). Therefore, bisulfite sequencing analysis may suggest that demethylation at the 24th and/or 31st CG plays an important role in the induction of AQP5 gene expression.

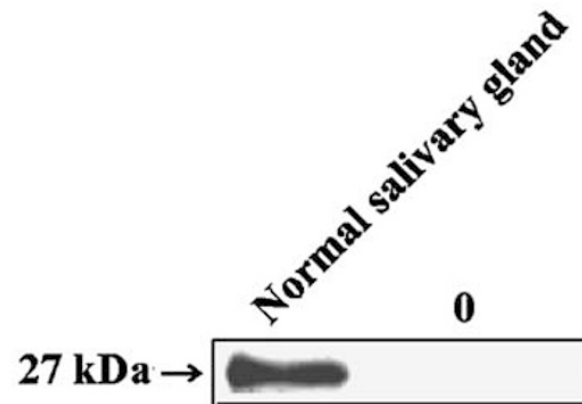

5-aza-2'-deoxycytidine $(2 \mu \mathrm{M})$

48

72

96

120

(h)

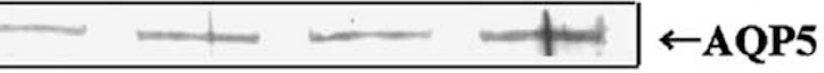

\section{AQP5/ $\beta$-actin ratio}

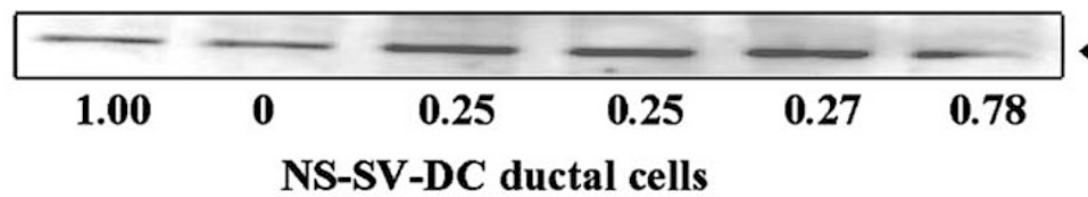

$\leftarrow \beta$-actin

\section{NS-SV-DC ductal cells}

Figure 5 Western blot analysis of AQP5 and $\beta$-actin proteins in crude plasma membranes from 5-Aza-CdR-treated NS-SV-DC ductal cells. Although the basal level of AQP5 expression $(0 \mathrm{~h})$ was not detected, increased expression of AQP5 protein with a molecular weight of $27 \mathrm{kDa}$ was clearly observed in NS-SV-DC cells after treatment with $2 \mu \mathrm{M} \mathrm{5}$-Aza-CdR (from 48 to $120 \mathrm{~h}$ of treatment). Densitometric analysis (AQP5/ $\beta$-actin ratio) revealed the expression level of AQP5 protein in 5-Aza-CdR-treated NS-SV-DC cells relative to that of normal salivary gland. These results were similar to those observed with quantitative real-time RT-PCR analysis. 
A

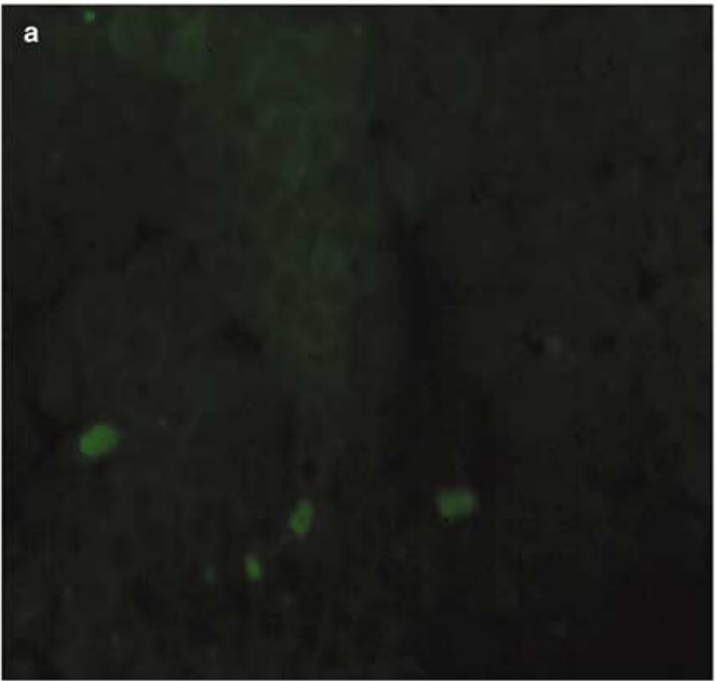

b

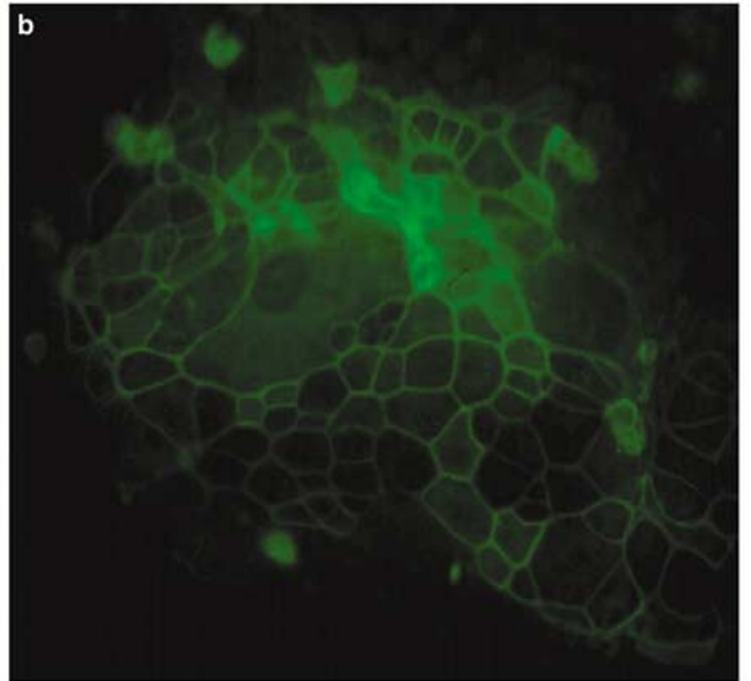

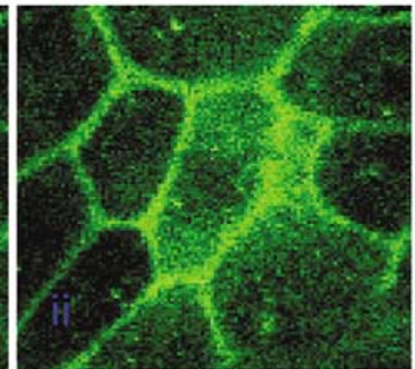
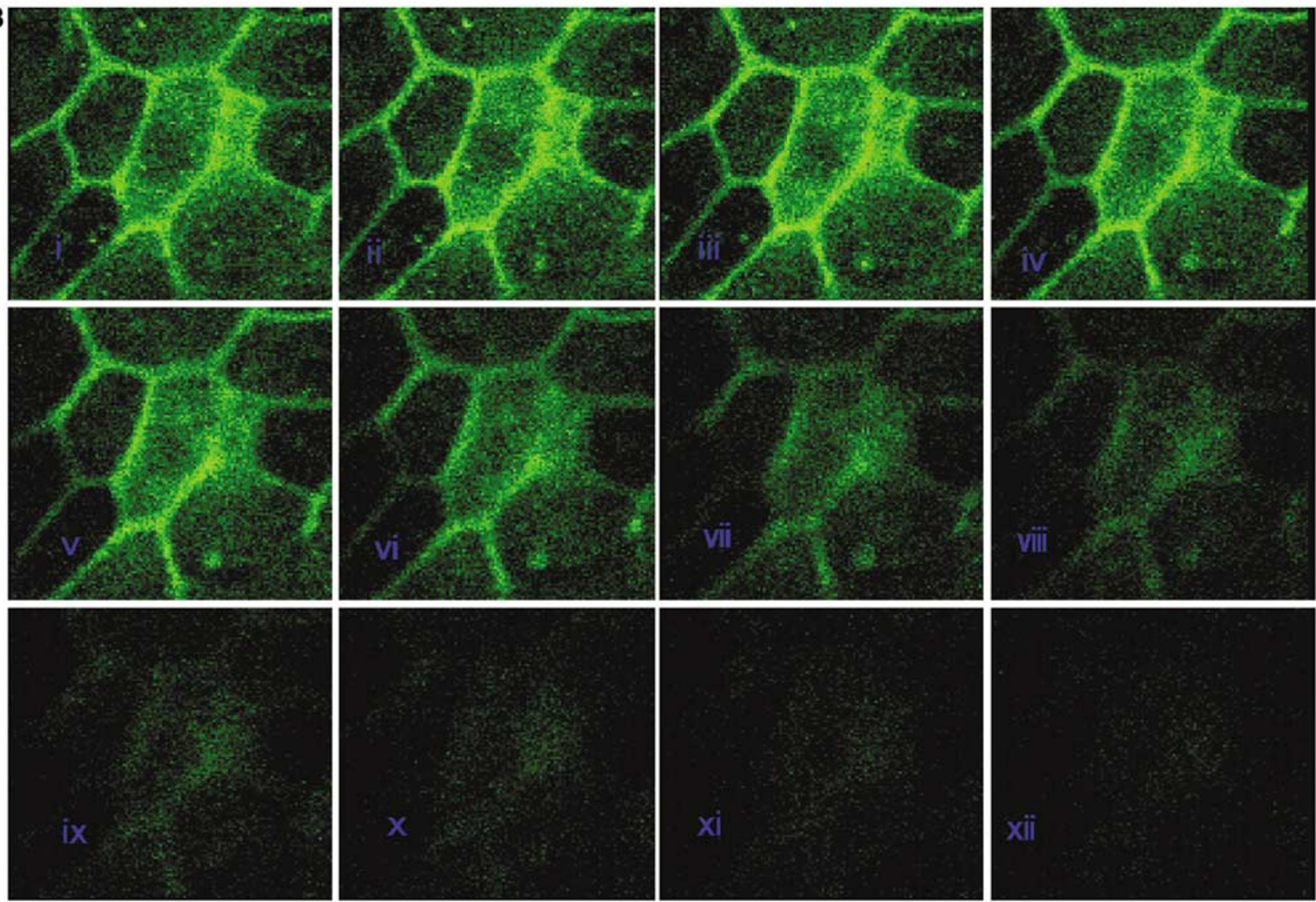

Figure 6 Localization of AQP5 in NS-SV-DC ductal cells. NS-SV-DC cells, grown on coverglasses, were treated with 5-Aza-CdR ( $2 \mu \mathrm{M})$ for $72 \mathrm{~h}$. The cells were then labeled for AQP5 and examined by confocal laser microscopy as described in Materials and methods. Micrographs of horizontal (xy; A, a and b), and vertical (serial optical sections of $0.7 \mu \mathrm{m}$ thickness from the apical surface (i) toward the basal surface (xii)) (xz; i-xii) optical images are shown. AQP5 staining at the apical and lateral sides of the plasma membrane can be seen in 5-Aza-CdR-treated NS-SV-DC cells (A, b and B, i-xii). However, no apparent staining for AQP5 was observed in untreated NS-SV-DC cells (A, a).

\section{Enhanced Transcriptional Activity of AQP5 Gene Promoter with Demethylation at the 24th and 31st CGs}

To demonstrate whether or not 5-Aza-CdR induces AQP5 expression through demethylation at the 24th and/or 31st CGs, luciferase vectors, whose methyla- tion status differed between the 24th and 31st CGs, were transiently transfected into NS-SV-DC cells to detect relative luciferase activity. As shown in Figure 9a, NS-SV-DC cells were transfected with a wild-type AQP5 promoter-luciferase fusion plasmid, pC3-Luc (methylated at all CGs including the 


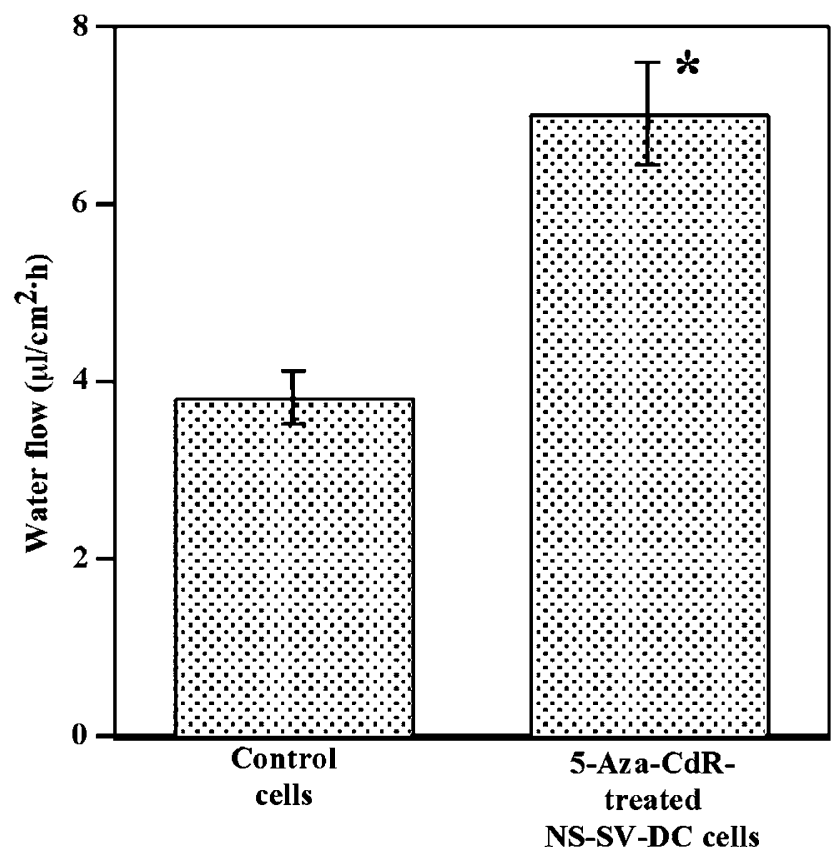

Figure 7 Net fluid secretion rates across NS-SV-DC monolayers. Net fluid secretion rates of control (untreated NS-SV-DC cells) and 5-Aza-CdR $(2 \mu \mathrm{M})$-treated NS-SV-DC cells were measured using a hyperosmotic medium (400 mOsm) at the apical side and isosmotic medium at the basolateral side. The results are expressed as fluid flow in $\mu \mathrm{l}$ secreted $/ \mathrm{cm}^{2} / \mathrm{h}$ and are the means \pm s.d. of three separate experiments performed in triplicate. The results were analyzed using the Mann-Whitney $U$-test. ${ }^{*} P<0.05$ compared with control cells.

1st, 23rd, 24th, 31st, and 33rd); with pD3-Luc (methylated at the 1st, 23rd, 31st, and 33rd CGs, and unmethylated at the 24th); with pD5-Luc (methylated at the 1st, 23rd, and 33rd CGs, and unmethylated at the 24th and 31st); or with pD7-Luc (methylated at the 1st, 23rd, 24th, and 33rd CGs, and unmethylated at the $31 \mathrm{st}$ ). The cells were then harvested at $24 \mathrm{~h}$ to measure the relative luciferase activity. As shown in Figure 9b, although the relative luciferase activity in wild-type pC3-Luctransfected and empty-vector-transfected NS-SV-DC cells was extremely low, pD3-Luc-transfected and pD7-Luc-transfected cells demonstrated significantly higher luciferase activity. Notably, when the pD5-Luc vector, which contains demethylated CGs at the 24th and 31st positions in the AQP5 promoter, was transfected into NS-SV-DC cells, the relative luciferase activity showed an additive effect of transfection with pD3-Luc or pD7-Luc alone. Accordingly, demethylation by 5-Aza-CdR at the 24th and 31st CGs in the AQP5 promoter may cooperatively function to induce AQP5 gene expression in NS-SV-DC ductal cells.

\section{Discussion}

Although the causes of xerostomia observed in patients with SS are unknown, the characteristic features of affected salivary glands in SS include various degrees of acinar destruction and the survival of ductal cells..,25,26 Thus far, we have shown, using an in vitro system, that the destruction of the acinar structure may be mediated in part by the disruption of the basement membrane's integrity through the increased production of matrix metalloproteinase (MMP)-9 from acinar (NS-SV-AC) cells; this increased production results from the cytokineinduced activation of the transcription factor NF$\kappa \mathrm{B}^{27}$ Another study showed that the inhibition of tumor necrosis factor- $\alpha$ (TNF- $\alpha$ )-induced activation of NF- $\kappa \mathrm{B}$ in acinar (NS-SV-AC) cells confers those cells with an ability to survive on the type IV collagen substrate. ${ }^{28}$ Taken together, these findings may indicate that anticytokine therapy targeted for the inhibition of $\mathrm{NF}-\kappa \mathrm{B}$ activity would contribute significantly to the interruption of the disease progression. However, one might speculate that this anticytokine therapy does not necessarily increase the fluid secretion from salivary glands, because this therapy only prevents the destruction of the acinar structure. Therefore, the aim of the present study was to investigate the possibility that surviving and/ or proliferating ductal cells in SS salivary glands could acquire a fluid-secreting function by expressing AQP5. We found that the treatment of ductal (NS-SV-DC) cells, which lack AQP5 expression, with the DNA demethylating agent 5-Aza-CdR results in the induction of AQP5 expression, thereby leading to increased fluid secretion.

Recent studies demonstrated that in SS salivary glands reduction in salivary flow is due to functional inhibition of salivary glands rather than to destruction of the glands by $\mathrm{T}$ lymphocytes. This is because a poor correlation was found between the degree of glandular destruction and the degree of dysfunction. ${ }^{29}$ Indeed, although the loss of acinar structure caused by infiltrated $\mathrm{T}$ lymphocytes directly reflects the decrease in salivary flow, it has been reported that cytokines, including TNF- $\alpha$ and interferon- $1 \beta$, secreted by $\mathrm{T}$ lymphocytes inhibit AQP5 expression by activating $\mathrm{NF}-\kappa \mathrm{B}$ activity in lung epithelial cells. ${ }^{30}$ This suggests that cytokines play an essential role in the pathogenesis of $\mathrm{T}$ lymphocyte-infiltrated salivary gland lesions in SS, and that anticytokine therapy is useful for the maintenance of AQP5 expression in SS acinar cells.

The accumulated evidence indicates that the localization of AQP5 is restricted to the apical membrane of acinar cells and to the proximal segment of intercalated duct cells in rat salivary glands. ${ }^{6,31-33}$ However, in our present study, expression of AQP5 was detected only in acinar (NS-SVAC) cells and not in ductal (NS-SV-DC) cells, indicating that NS-SV-DC cells originate from striated or excretory ducts. Recently, high-resolution confocal microscopy and quantitative image analysis in human salivary glands have found AQP5 expression in acinar cells only. ${ }^{34}$ Thus, subcellular localization of AQP5 may be species-specific. 
a AAGGGGAACC CCGGCCTGGG AGAGGGCGCC TCCGGGGATC $\stackrel{1}{\text { CGTTGCCTAG TCCAGGTACT }}$ Sp1-1

$\begin{array}{llllll}5 & 6 & 7 & 8 & 9 & 10\end{array}$

11

GCCCAGCTAC CGGGCGTCGA GGATTGCGAA CGGTCGGGGC AGGCTGGCAC GGTGCCCACT

TTTCCCAAAA CTCCAGCCTT CCAAGCCCAG AAGCTCGCCC GGCCCAGGCC GAGCTGGCCA

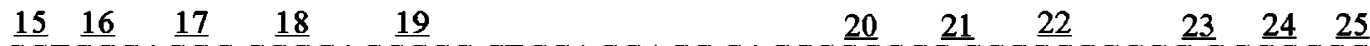

CGTCGGACGG CCGGACCGCC CTGCAGGACC CAGCCCGGCC GCCCGCCCC GCEGGCGGTG Sp1-2

$\begin{array}{llllll}26 & 27 & 28 & 29 & 30 & 31\end{array}$

AGGGAGGTGA GCGGCGCCGA CCTGCGGGAC GAGCATCACT CCGACCCAGC CGGGGGTGAG

$$
\underline{37} \quad \underline{38} \quad \underline{39} \quad \underline{40} \quad \underline{41}
$$
$42 \quad 43 \longrightarrow$ Transcription start site GAAGAAAAGG GGAAGGCCGC GCACGGAGCG CGGTAAAGGC CGGCGGAGCT AGACGCCCCG +14

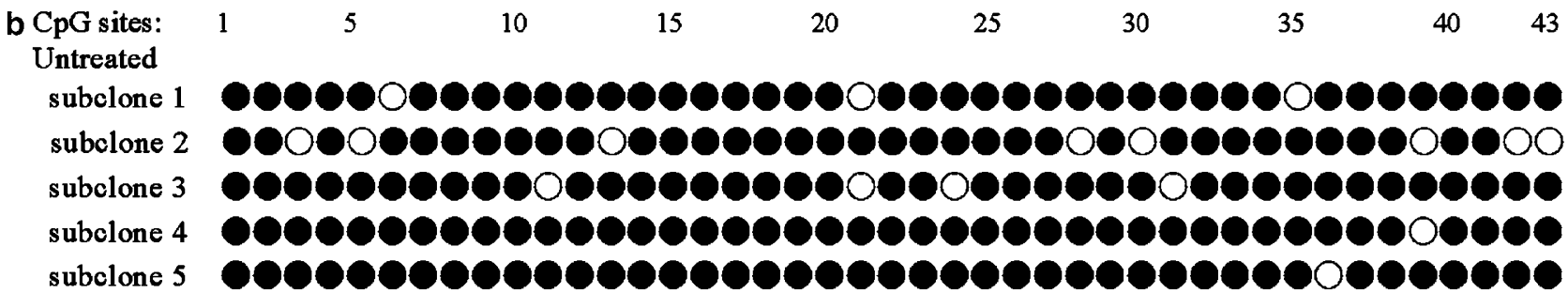

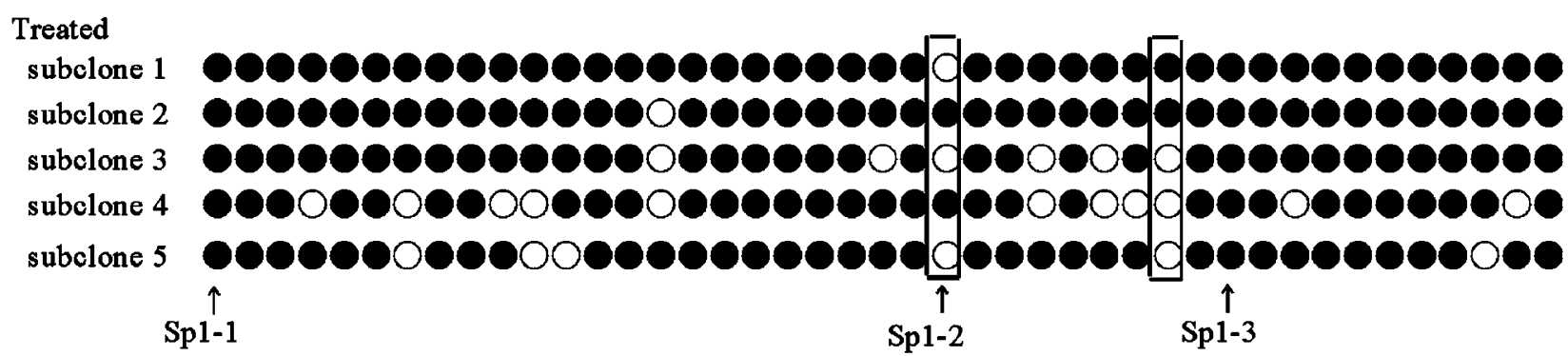

Figure 8 Bisulfite sequencing of CpG island in the AQP5 promoter. (a) The CpG island of the AQP5 promoter (GenBank ${ }^{\mathrm{TM}} / \mathrm{EMBL}$ Data Bank no. U46566) was analyzed. This sequence spans 578 bp between positions -406 to +172 relative to the transcription start site, including 43 CGs upstream of the transcriptional start site. Three CG-containing Sp1-binding sites within this sequence are indicated as underlined and emboldened, corresponding to the 1st, 23rd, 24th, and 33rd CGs within this island. (b) DNA from control NS-SV-DC and 5-Aza-CdR ( $2 \mu \mathrm{M})$-treated NS-SV-DC cells was treated with bisulfite, and the AQP5 promoter was PCR amplified. The PCR product was ligated into pCR2.1-TOPO by using the TA cloning system. Five subclones, each from control cells, and 5-Aza-CdR-treated cells were picked and sequenced. Symbols: $\bigcirc$, unmethylated cytosines; $\bullet$, methylated cytosines. Demethylation was observed at the CGs both in the second Sp1 and around the third Sp1 sites, as indicated by boxes.

Although a strong correlation between promoter methylation and gene silencing has been extensively demonstrated, ${ }^{35-37}$ the molecular mechanism of this methylation-modulated gene inactivation remains unclear. Among the proposed hypotheses regarding the transcriptional inactivation from promoter methylation, ${ }^{38-42}$ one hypothesis that gene repression is induced by a direct influence of methylation on the binding of transcription factors may be related to the results obtained in our study. Since many mammalian transcription factors have CGrich-binding sites in their DNA recognition elements, methylation occurring at the CG of a specific binding element may sterically interfere with the binding of transcription factors to DNA, thus inhibiting transcription. ${ }^{36}$ For instance, an HpaII site (CCGG) is located in an element to which the transcription factor AP-2 binds. Methylation at this 


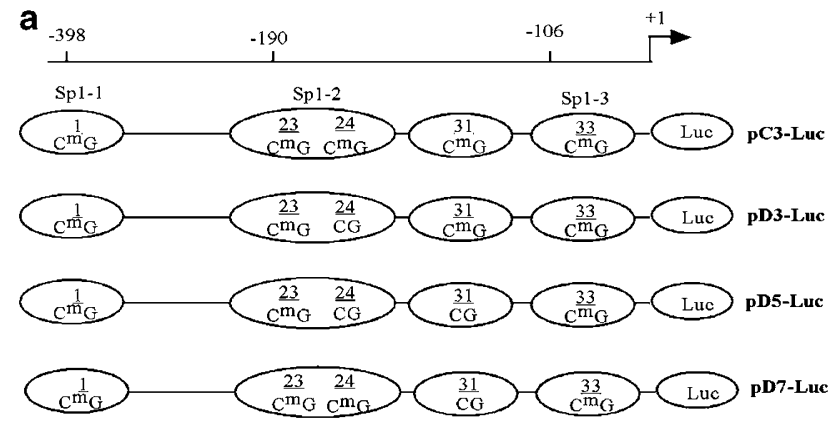

b

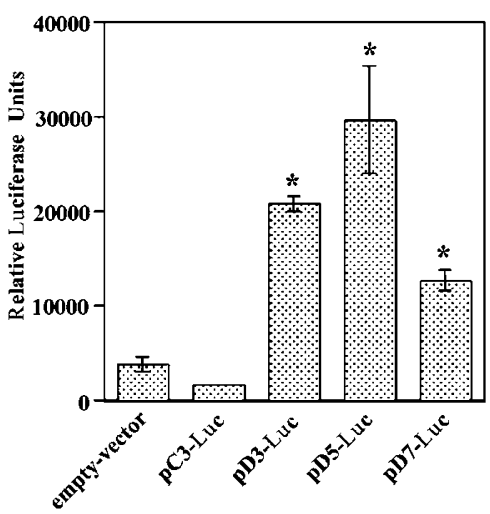

Figure 9 Analysis of relative luciferase activity in NS-SV-DC cells. (a) AQP5-promoter constructs used for the luciferase transfection assay. The human wild-type AQP5 promoter luciferase fusion plasmid, pC3-Luc, contains all methylated CGs, including the 1st, 23rd, 24th, 31st, and 33rd, as well as the transcription start site. pD3-Luc contains an unmethylated CG at the 24th position and methylated CGs at the 1st, 23rd, 31st, and 33rd positions. pD5-Luc contains unmethylated CGs at the 24th and 31st positions and methylated CGs at the 1st, 23rd, and 33rd positions. pD7-Luc contains an unmethylated CG at the 31st position and methylated CGs at the 1st, 23rd, 24th, and 33rd positions. (b) At $24 \mathrm{~h}$ after transfection, NS-SV-DC cells were harvested for analysis of luciferase activity. The luciferase activity of each sample was normalized for the amount of protein in the cell lysate. The experiments were carried out at least two times in triplicate. The luciferase activity of NS-SV-DC cells transfected with an empty vector served as a control. A significant increase in the luciferase activity was detected when pD3-Luc or pD7-Luc vector alone was transfected into NS-SV-DC cells. In addition, transfection of pD5-Luc vector showed an additive effect of both pD3-Luc and pD7-Luc on the increase in luciferase activity in NS-SV-DC cells. The results were analyzed using Mann-Whitney $U$-test. ${ }^{*} P<0.05$ compared with empty-vector-transfected and pC3-Luc-transfected cells.

HpaII site (CC ${ }^{\mathrm{m}} \mathrm{GG}$ ), within the AP-2-binding site, inhibits AP-2 binding and suppresses AP-2-regulated gene transcription in C6-glioma and CV-1 cells. ${ }^{43}$ This direct effect of methylation on the binding of transcription factors is also observed in other genes, including cyclic AMP (cAMP)/cAMPresponsive element (TGACGTCA) ${ }^{44}$ and the retinoblastoma binding factor 1 recognition sequence (AGCTGCCGCGGGCGGAAGT). ${ }^{45}$ However, reports regarding the effect of methylation at the Sp1binding site on Sp1 binding are conflicting. In fact, although several researchers have shown that methylation at Sp1-binding sites has no influence on Sp1 binding or gene expression, ${ }^{45,46}$ others have reported that methylated CpG dinucleotides variably interfere with Sp1 binding. ${ }^{47,48}$ Consistent with the latter finding, our present study demonstrated that methylation at the Sp1-binding site (24th CG) of the AQP5 promoter partially suppressed the transcriptional activity of the AQP5 gene. Thus, which mechanism is predominant in the methylation-induced gene repression may depend on the cell type, transcription factor, or received stimuli. ${ }^{49}$

Our study also showed that demethylation at the CG site (31st CG) outside of the third consensus Sp1 element of the AQP5 promoter significantly induced the transcriptional activity of the AQP5 gene. The exact mechanism by which methylation suppresses gene expression at CGs outside of consensus DNAbinding sites is not clear. However, similar results have recently been reported by other investigators, that is, the $5^{\prime}$-flanking region of cyclin D1 in rat leukemia cell lines was found to be methylated around two continuous Sp1-binding sites in the cyclin D promoter. Methylation that was not within but adjacent to the two Sp1 sites in this promoter significantly reduced cyclin D expression. ${ }^{50}$ That study indirectly showed that, at the CG outside the Sp1 element, methylation might have played a critical role in reducing gene expression. Moreover, EMSA experiments have produced direct evidence that methylation at CGs outside the consensus Sp1 site reduces $\mathrm{Sp} 1$ binding and gene expression in the $\mathrm{p} 21^{\mathrm{Cip} 1}$ gene promoter. ${ }^{49}$ Thus, it may be evident that methylation outside of a transcription factor's cognate recognition sequence can affect the transcription factor's DNA-binding activity, and that inhibition of this methylation is correlated with reexpression of the relevant gene.

In conclusion, the results of this study indicate that demethylation by 5-Aza-CdR functions between the two CG sites cooperatively in the AQP5 promoter to induce AQP5 gene expression in human salivary gland ductal cells. Although our results may appear to lack overt exocrine physiological relevance, they do suggest that it is possible for cells with ductal phenotypes to express functional water channels and exhibit considerable water permeability. Thus, 5-Aza-CdR treatment of SS salivary glands should provide an efficient and useful means to impart facilitated water permeability to surviving and/or proliferating ductal cells in SS salivary glands. This possibility seems to be worthy of further investigation.

\section{Acknowledgements}

This work was supported by a Grant-in-Aid from the Ministry of Education, Science, Sports, and Culture of Japan. 


\section{References}

1 Alpaugh MA, Whaley WW, Wohl MJ, et al. Sjögren's syndrome. In: Kekkey WN, Harris Jr ED, Ruddy S, Sledge CB (eds). Textbook of Rheumatology. WB Saunders: Philadelphia, 1981, pp 971-999.

2 Daniel TE. Labial salivary gland biopsy in Sjögren's syndrome: assessment as a diagnostic criterion in 362 suspected cases. Arthritis Rheum 1984;27:147-156.

3 Rosai J. Major and minor salivary glands. In: Rosai J (ed). Ackerman's Surgical Pathology. Mosby-Year Book: New York, 1996, pp 815-818.

4 King LS, Yasui M. Aquaporins and disease: lessons from mice to humans. Trends Endocrinol Metab 2002;13:355-360.

5 Raina S, Preston GM, Guggino WB, et al. Molecular cloning and characterization of an aquaporin cDNA from salivary, lacrimal, and respiratory tissues. J Biol Chem 1995;270:1908-1912.

6 Agre P, Brown D, Nielsen S. Aquaporin water channels: unanswered questions and unresolved controversies. Curr Opin Cell Biol 1995;7:472-483.

7 Ma T, Song Y, Gillespie A, et al. Defective secretion of saliva in transgenic mice lacking aquaporin-5 water channels. J Biol Chem 1999;274:20071-20074.

8 Gresz V, Kwon TH, Hurley PT, et al. Identification and localization of aquaporin water channels in human salivary glands. Am J Physiol 2001;281:G247-G254.

9 Laird PW, Jaenisch R. The role of DNA methylation in cancer genetics and epigenetics. Annu Rev Genet 1996; 30:441-464.

10 Baylin SB, Herman JG, Graff JR, et al. Alterations in DNA methylation: a fundamental aspect of neoplasia. Adv Cancer Res 1998;72:141-196.

11 Jones PA, Laird PW. Cancer epigenetics of age. Nat Genet 1999;21:163-167.

12 Di Croce L, Raker VA, Corsaro M, et al. Methyltransferase recruitment and DNA hypermethylation of target promoters by an oncogenic transcription factor. Science 2002;295:1079-1082.

13 Cameron EE, Bachman KE, Myohanen S, et al. Synergy of demethylation and histone deacetylase inhibition in the re-expression of genes silenced in cancer. Nat Genet 1999;21:103-107.

14 Daskalakis M, Nguyen TT, Nguyen C, et al. Demethylation of a hypermethylated P15/INK4B gene in patients with myelodysplastic syndrome by 5 -aza-2'-deoxycytidine (decitabine) treatment. Blood 2002;100:29572964.

15 Kaneda A, Kaminishi M, Yanagihara K, et al. Identification of silencing of nine genes in human gastric cancers. Cancer Res 2002;62:6645-6650.

16 Zhu B, Chen Z, Cheng X, et al. Induction of TRAG-3 expression in A549 lung adenocarcinoma cell line by 5 -aza-2'-deoxycytidine. Lung Cancer 2002;38: 321-322.

17 Chen H, Toyooka S, Gazdar AF, et al. Epigenetic regulation of a novel tumor suppressor gene (hDAB2IP) in prostate cancer cell line. J Biol Chem 2003;278:3121-3130.

18 Lee MD, Bhakta KY, Raina S, et al. The human aquaporin-5 gene. J Biol Chem 1996;271:8599-8604.

19 Azuma M, Tamatani T, Kasai Y, et al. Immortalization of normal human salivary gland cells with duct-, myoepithelial-, acinar-, or squamous phenotype by transfection with SV40 ori-mutant DNA. Lab Invest 1993;69:24-42.
20 Azuma M, Motegi K, Aota K, et al. Role of cytokines in the destruction of acinar structure in Sjögren's syndrome salivary glands. Lab Invest 1997;77:269-280.

21 Neufeld TK, Grant ME, Grantham JJ. A method to measure the rate of net fluid secretion by monolayers of cultured renal epithelial cells. J Tissue Cult Methods 1991;13:229-234.

22 Delporte C, O’Connell BC, He X, et al. Adenovirusmediated expression of aquaporin-5 in epithelial cells. J Biol Chem 1996;271:22070-22075.

23 Esteller M, Sparks A, Toyota M, et al. Analysis of adenomatous polyposis coli promoter hypermethylation in human cancer. Cancer Res 2000;60:4366-4371.

24 Huang L, Sowa T, Sakai T, et al. Activation of the p21WAF1/CIP1 promoter independent of p53 by the histone deacetylase inhibitor suberoylanilide hydroxamic acid (SAHA) through the Sp1 sites. Oncogene 2000;19:5712-5719.

25 Takeda Y, Komori A. Focal lymphocytic infiltration in the human labial salivary glands: a postmortem study. J Oral Pathol 1985;15:83-86.

26 Delporte CB, O’Connell C, He X, et al. Increased fluid secretion after adenoviral-mediated transfer of the aquaporin-1 cDNA to irradiated rat salivary glands. Proc Natl Acad Sci USA 1997;94:3268-3273.

27 Azuma M, Aota K, Tamatani T, et al. Suppression of TNF- $\alpha$-induced MMP-9 production by the introduction of super-repressor form of $\mathrm{I} \kappa \mathrm{B} \alpha \mathrm{cDNA}$ into immortalized human salivary gland acinar cells. Arthritis Rheum 2000;43:1756-1767.

28 Azuma M, Aota K, Tamatani T, et al. Suppression of TNF- $\alpha$-induced MMP-9 production in human salivary gland acinar cells by cepharanthine occurs via down-regulation of NF- $\kappa$ B. Arthritis Rheum 2002; 46:1585-1594.

29 Humphreys-Beher MG, Brayer J, Yamachika S, et al. An alternative perspective to the immune response in autoimmune exocrinopathy: induction of functional quiescence rather than destructive autoaggression. Scand J Immunol 1999;49:7-10.

30 Towne JE, Krane CM, Bachurski CJ, et al. Tumor necrosis factor-alpha inhibits aquaporin 5 expression in mouse lung epithelial cells. J Biol Chem 2001; 276:18657-18664.

$31 \mathrm{He}$ X, Tse C-M, Donowitz M, et al. Polarized distribution of key membrane transport proteins in the rat submandibular gland. Pflugers Arch 1997;433: 260-268.

32 Nielsen S, King LS, Christensen BM, et al. Aquaporins in complex tissues. II. Subcellular distribution in respiratory and glandular tissues of rat. Am J Physiol 1997;273:C1549-C1561.

33 Matsuzaki T, Suzuki T, Koyama H, et al. Aquaporin-5 (AQP5), a water channel protein, in the rat salivary and lacrimal glands: immunolocalization and effect of secretory stimulation. Cell Tissue Res 1999;295: $513-521$

34 Beroukas D, Hiscock J, Jonsson R, et al. Subcellular distribution of aquaporin 5 in salivary glands in primary Sjögren's syndrome. Lancet 2001;358:18751876.

35 Jones P, Takai D. The role of DNA methylation in mammalian epigenetics. Science 2001;293:1068-1070.

36 Bird A. DNA methylation patterns and epigenetic memory. Genes Dev 2002;16:6-21.

37 Plass C, Soloway PD. DNA methylation, imprinting, and cancer. Eur J Hum Genet 2002;10:6-16. 
38 Tate PH, Bird AP. Effects of DNA methylation on DNAbinding proteins and gene expression. Curr Opin Genet Dev 1993;3:226-231.

39 Cross SH, Charlton JA, Nan X, et al. Purification of CpG islands using a methylated DNA binding column. Nat Genet 1994;6:236-244.

40 Bird AP, Wolffe AP. Methylation-induced repression: belts, braces, and chromatin. Cell 1999;99:451-454.

41 Robertson KD, Jones PA. DNA methylation: past, present, and future directions. Carcinogenesis 2000;21: 461-467.

42 Rountree MR, Bachman KE, Herman JG, et al. DNA methylation, chromatin inheritance, and cancer. Oncogene 2001;20:3156-3165.

43 Comb M, Goodman HM. CpG methylation inhibits proenkephalin gene expression and binding of the transcription factor AP-2. Nucleic Acid Res 1990;18: 3975-3982.

44 Iguchi-Ariga SM, Schaffner W. CpG methylation of the cAMP-responsive enhancer/promoter sequence TGACGTCA abolishes specific factor binding aswell as transcriptional activation. Genes Dev 1998;3: 612-619.
45 Ohtani-Fujita N, Fujita T, Aoike A, et al. CpG methylation inactivates the promoter activity of the human retinoblastoma tumor-suppressor gene. Oncogene 1993;8:1063-1067.

46 Holler M, Westin G, Jiricny J, et al. Sp1 transcription factor binds DNA and activates transcription even when the binding site is CpG methylated. Genes Dev 1988;2:1127-1135.

47 Clark SJ, Harrison J, Molloy PL. Sp1 binding is inhibited by $(\mathrm{m}) \mathrm{Cp}(\mathrm{m}) \mathrm{CpG}$ methylation. Gene 1997; 195:67-71.

48 Mancini DN, Singh SM, Archer TK, et al. Site-specific DNA methylation in the neurofibromatosis (NF1) promoter interferes with binding of CREB and SP1 transcription factors. Oncogene 1999;18:4108-4119.

49 Zhu W-G, Srinivasan K, Dai Z, et al. Methylation of adjacent $\mathrm{CpG}$ sites affects Sp1/Sp3 binding and activity in the p21Cip1 promoter. Mol Cell Biol 2003; 23:4056-4065.

50 Kitazawa S, Kitazawa R, Maeda S. Transcriptional regulation of rat cyclin D1 gene by CpG methylation status in promoter region. J Biol Chem 1999;274: 28787-28793. 